\title{
Conséquence de la sécheresse sur l'alimentation en eau potable. Aspect quantitatif
}

\author{
P. Bichon \\ Compagnie générale des eaux, Paris
}

\section{Introduction}

La sécheresse se caractérisant essentiellement par un déficit de la pluviométrie par rapport à celle de la période normale correspondante, pendant une durée plus ou moins longue, il semble évident qu'elle va poser des problèmes aux services chargés de l'alimentation en eau potable dont la matière première provient uniquement des précipitations.

Ces problèmes seront d'autant plus aigus que pendant la durée de la sécheresse les besoins en eau potable auront tendance à s'accroître puisque le service public devra d'abord répondre aux besoins habituels, en général accrus à cause de la canicule qui accompagne habituellement la sécheresse, mais aussi pour se substituer aux ressources extérieures qui vont peu à peu se tarir : puits des particuliers, citernes d'eau de pluie, et enfin pour assurer la desserte de besoins nouveaux engendrés par la sécheresse ou la chaleur ambiante: arrosages inhabituels de jardins ou même de toitures.

Ainsi, en plus des soucis de qualité d'eau distribuée dus essentiellement à la concentration accrue d'un flux de pollution inchangé dans des cours d'eau au débit réduit, le distributeur d'eau va se trouver confronté aux problèmes de quantités à fournir, théoriquement accrues, à partir d'une ressource défaillante, ou tout au moins mal ou pas alimentée. C'est de cet aspect quantitatif qu'il va être traité dans le présent exposé.

\section{Deux caractéristiques de la sécheresse}

Cet exposé relatif aux problèmes quantitatifs créés par la sécheresse aux distributeurs d'eau met en relief deux caractéristiques présentées par le phénomène.

a) D'une part, parmi les fléaux naturels, celui de la sécheresse se manifeste progressivement. Ce n'est pas comme un tremblement de terre, ou un violent orage, ou un cyclone, ou même certaines inondations, qui, même si ces derniers phénomènes peuvent se prévoir plusieurs jours à l'avance, fondent brutalement sur les régions concernées, ne laissant pas aux responsables le temps de prendre des mesures appropriées.

Au contraire, une période de sécheresse se prévoit et se prépare : s'il est impossible de prévoir que l'année à venir sera particulièrement sèche, en revanche la période de sécheresse ne commencera à se manifester qu'après plusieurs mois de déficit pluviométrique pendant lesquels on pourra commencer à prévoir les mesures à prendre; et ces mesures elles-mêmes pourront s'étaler dans le temps au fur et à mesure que la sécheresse s'aggravera ; inversement si des précipitations viennent mettre un terme à la prévision de sécheresse ou à la sécheresse elle-même il sera possible de surseoir aux mesures envisagées.

b) Une deuxième caractéristique du phénomène sécheresse vis-à-vis de la distribution d'eau, c'est l'extrême diver-

\section{Consequences of the drought on supply of drinking water. Quantitative aspect}

The drought of 1976 was felt particularly hard in the west of France. In these regions where underground nappes are rare, the water services are mainly supplied from waterways or reservoirs created for this purpose. We will examine the effect of drought on supplies for the main towns in the west and on the different sized services of this region, according to whether they are supplied by surface water or underground water. Since the drought led to shortages of supplies, we will examine the steps taken firstly to reduce demand: restriction of needs, prevention of waste, regulatory measures, then how to find emergency supplies, or transport by temporary channels or by road. Finally we will draw some conclusions for future installations. 
sité de son impact sur les différents services d'eau d'un même pays. Et ceci pour plusieurs raisons :

D'abord parce que les différentes régions ne sont pas affectées de la même façon par les déficits pluviométriques : on verra dans les exemples étudiés ci-après qu'une sécheresse peut toucher plusieurs pays mais qu'elle se manifestera de façon très différente d'une région à l'autre, par l'intensité des déficits, par leur étalement dans le temps, par les dates de reprise des précipitations normales.

Ensuite, dans les régions touchées, l'impact du phénomène est variable en fonction du type de prélèvement dans le milieu naturel de l'eau alimentant le service. Le service s'alimentant dans une nappe profonde n'en souffrira pas alors qu'un service puisant dans la nappe phréatique verra sa ressource diminuer dangereusement ou même se tarir. Tel service prélevant dans un barrage mal alimenté pourra manquer d'eau tandis qu'un autre pompant dans une rivière pourra desservir ses abonnés tant que la rivière coulera.

Nous allons examiner ces deux caractéristiques à la lumière de ce qui s'est passé en France pendant la sécheresse exceptionnelle de 1976.

\section{Sécheresse de 1976 : progressivité du phénomène}

Ce qui suit est extrait d'un rapport établi en 1977 par la Mission interministérielle de l'eau, qui a assuré au niveau national la coordination des actions de l'Administration pendant la sécheresse de 1976 :

«Du point de vue pluviométrique, l'année 1976 est caractérisée par une sécheresse exceptionnelle. La durée de retour d'une telle situation est dans les régions les plus touchées d'ordre séculaire. "

"On peut fixer le début de l'épisode de sécheresse au mois de décembre 1975. A compter de ce mois, et à l'exception des régions méditerranéennes et pyrénéennes où les déficits ont été globalement soit faibles, soit inexistants, la plupart des mois de l'hiver 1975-1976, du printemps et du début de l'été 1976 sont caractérisés par une pluviométrie déficitaire. Pendant certains mois on a recueilli des hauteurs de précipitation exceptionnellement faibles (décembre, janvier, mars, mai et surtout juin). ”

"C'est cette succession de mois déficitaires en pluie entre décembre et août, associée à des séquences anormales de journées très chaudes en juin et juillet qui a donné un caractère véritablement exceptionnel à cette sécheresse. "

On voit bien ici le caractère progressif de l'apparition du phénomène. Le rapport situe le début de l'épisode au mois de décembre. Les distributeurs ont commencé à s'intéresser au phénomène en mars-avril, à envisager des mesures, là où elles étaient nécessaires, en avril-mai et à mettre en application ces mesures en mai-juin et juillet, alors même que les conséquences directes de cette sécheresse, pourtant amorcée dès décembre 75 , ne commençaient pas à se manifester, sur les services les plus touchés, avant mai 1976.

\section{Le rapport poursuit :}

"A titre indicatif, le rapport pluviométrique - c'està-dire le rapport des pluies tombées entre le $1^{\text {er }}$ décembre 1975 et le 31 juillet 1976 aux pluies moyennes de la même période pour les années 1931-1960 - a été inférieur :

- à 0,40 dans la majeure partie de la Bretagne, de la Normandie, des Pays de Loire, de la Picardie, de la Champagne, du Limousin et certaines zones de l'Ile-de-France ; - à 0,60 dans pratiquement toute la moitié nord de la France. 》

On voit sur ce $2^{\mathrm{e}}$ extrait qu'une partie du Pays a été plus touchée que l'autre. Il faut ajouter que les pluies mettant fin à la sécheresse ont commencé à tomber dès la fin du mois de mai dans le midi de la France qui n'a donc pratiquement pas subi les conséquences de la sécheresse.

\section{Diversité des situations}

Les services d'eau sont de la compétence des communes. Cela tient à des raisons historiques d'autonomie communale, chaque agglomération faisant son affaire de distribuer à ses habitants l'eau extraite localement du milieu naturel. Et puis l'on s'est rendu compte qu'amener l'eau dans une autre agglomération voisine non desservie coûterait plus cher que de créer un $2^{\mathrm{e}}$ service propre à cette agglomération. Les réseaux ne se sont donc pas étendu. La législation concernant les services d'eau s'est développée en réglementant ce qui existait. Les communes ont cependant la possibilité de se regrouper en Syndicat lorsque les conditions d'établissement d'un service commun se révèlent plus intéressantes.

De cette façon chacune des collectivités locales (commune ou Syndicats de commune) dispose d'un service d'eau indépendant avec ses installations propres de production (pompage et éventuellement traitement) et son réseau de distribution limité à son territoire et sans canalisations de liaison avec les réseaux voisins.

En fonction du type de prélèvement dans la ressource, le service sera plus ou moins vulnérable à une sécheresse comme celle de 1976.

Les nappes profondes sont moins sensibles à la plus ou moins grande importance des précipitations de l'année. Les nappes phréatiques, à l'opposé, sont très vulnérables au déficit des pluies: le niveau des nappes suit celui des précipitations. Enfin un troisième type de prélèvement est celui qui sollicite l'eau des cours d'eau ou leurs nappes d'accompagnement. Il est rare qu'un fleuve s'assèche dans nos régions mais les nappes alluviales peuvent voir leur niveau descendre au-dessous de celui d'aspiration des pompes. Le cas de l'eau prélevée dans les barrages de retenue est par lui-même très variable suivant le régime de fonctionnement de cette retenue.

La sécheresse de 1976 qui a frappé durement une grande partie de la France, et a eu des effets catastrophiques sur l'agriculture, a eu des impacts très divers sur les services d'alimentation en eau en fonction de leur type de prélèvement de l'eau dans la ressource. 


\section{Mesures à prendre}

Les différentes considérations ci-dessus expliquent les mesures que l'on peut être amené à prendre, progressivement en ce qui concerne les premières, préventivement pour les autres au fur et à mesure que les menaces se précisent.

Mesures de réduction de la demande. Elles peuvent être prises à différents échelons, par solidarité, mais ne se justifient qu'au niveau des services touchés :

- limitation ou interdiction de certains usages privés de l'eau: arrosage des jardins, lavage des voitures;

- limitation ou arrêt de certains usages publics : lavage des rues, alimentation de fontaines publiques, allongement des périodes de chasses d'agouts;

- campagnes d'information de la population avec recommandations de limiter les tirages, particuliers ou collectifs ou industriels ;

- enfin coupures périodiques de la distribution. Cette mesure est celle de la dernière extrémité, en raison des inconvénients de toutes sortes que présentent des périodes de vidanges et de remises en eau de réseaux.

Mises en auvre de moyens de dépannage. Cela peut concerner l'accélération des travaux de renforcement déjà prévus mais non encore réalisés, mais cela concerne aussi des réalisations provisoires pour passer le cap difficile de l'insuffisance des ressources propres du service :

- dépannage par un service voisin mieux approvisionné. On a vu en 1976 allonger dans la campagne des kilomètres de canalisations provisoires pour réaliser des interconnexions entre services ;

- recherches d'eau brute inutilisée jusque-là : forage de puits supplémentaire, réutilisation de ressources abandonnées, utilisation de l'eau d'étangs ou de retenues non utilisées pour l'alimentation en eau ;

- cette dernière solution comme celle qui consiste à utiliser l'eau d'une rivière voisine (non asséchée...) impliquent soit qu'existent les moyens de traitement de ces eaux soit que soient mis en place des traitements provisoires. Ainsi au cours de l'été 1976 ont été utilisées un maximum de stations de traitement mobiles;

- enfin en cas d'impossibilité de réalimenter le service défaillant, il faudra alimenter les populations par des camions-citernes.
Nous ajouterons deux remarques concernant les solutions de dépannage :

- d'une part, la sécheresse ne touche pas que l'alimentation en eau des services de distribution, mais tous les autres utilisateurs, agriculteurs et industriels en particulier. Les restrictions obligent à donner un ordre de priorité aux utilisateurs, l'alimentation en eau de la population devant être considérée comme primordiale. Ce sont les préfets qui doivent rendre les arbitrages dans les conflits qui ne manquent pas de se produire ;

- d'autre part la pénurie de la ressource s'accompagne d'un accroissement de son degré de pollution. Si tout doit être mis en œuvre pour lutter contre les déficits en eau, il faudra être particulièrement vigilants sur les traitements à appliquer qui iront au-delà des cycles habituels ; toutefois on pourra être plus tolérant sur les caractéristiques de confort que sont les qualités organoleptiques par exemple, à condition d'être intransigeant sur les caractéristiques de potabilité, et en particulier de potabilité bactériologique.

\section{Circulaire du 8 septembre 1988 sur la sécheresse}

Les années ont passé, on peut penser que les services les plus touchés ont pris les mesures propres à éviter le retour des mêmes difficultés. De son côté, au moment où la Société hydrotechnique de France décidait de consacrer aux "Sécheresses" les présentes journées, le Secrétaire d'Etat à l'Environnement publiait la circulaire 88-75 du 8 septembre 1988 intitulée "Mesures de police et de gestion des eaux en période de sécheresse ».

Nous n'en ferons pas ici l'analyse, nous contentant de citer son premier alinéa :

«La présente circulaire a pour objet de rappeler les divers moyens dont dispose l'administration en période de sécheresse pour préserver l'alimentation en eau destinée à la consommation humaine. "

Il est bon qu'une telle circulaire ait été rédigée, évitant aux divers responsables d'être pris au dépourvu en cas de retour d'une nouvelle sécheresse. On peut cependant souhaiter en conclusion qu'elle ne trouve son application que dans un avenir le plus lointain possible.

Nota: Le texte ci-dessus a été modifié le jour de l'exposé pour s'appuyer sur des exemples pris dans l'actualité, puisqu'au mois de juin 1989, la sécheresse qui s'est amplifiée par la suite avait déjả commencé à se manifester. 